\title{
Effects of beta 2 adrenergic agonists on axonal injury and mitochondrial metabolism in experimental autoimmune encephalomyelitis rats
}

\author{
Z.W. Zhang ${ }^{1,2}$, X.Y. Qin', F.Y. Che ${ }^{2}$, G. Xie ${ }^{2}$, L. Shen ${ }^{2}$ and Y.Y. Bai ${ }^{2}$ \\ ${ }^{1}$ Department of Neurology, the First Affiliated Hospital of Chongqing Medical University, \\ Chongqing, China \\ ${ }^{2}$ Department of Neurology, Jiangjin Central Hospital, Chongqing, China \\ Corresponding author: Z.W. Zhang \\ E-mail: zhangzuowen201501@163.com \\ Genet. Mol. Res. 14 (4): 13572-13581 (2015) \\ Received April 23, 2015 \\ Accepted August 13, 2015 \\ Published October 28, 2015 \\ DOI http://dx.doi.org/10.4238/2015.October.28.17
}

ABSTRACT. The primary aims of this study were to investigate mitochondrial metabolism during experimental allergic encephalomyelitis (EAE) animal model axonal injury and to determine the correlation among neurological function scores, pathological changes, and the activities of the $B B$ isoenzyme of creatine kinase (CK-BB), catalase (CAT), and calpain in the brain tissues of EAE rats. Another goal was to preliminarily define the mechanism of mitochondrial metabolism resulting from the effect of beta 2 adrenergic agonists in the process of EAE animal model axonal damage. EAE was induced in specific pathogen free Wistar rats by guinea pig spinal cord homogenate, complete Freund's adjuvant, and pertussis vaccine. We recorded the behavioral change in EAE rats, detected pathological changes in central nervous tissue, and observed the changes of the CK$\mathrm{BB}, \mathrm{CAT}$, and calpain in the EAE rat brain and spinal cord. The results indicated that the average neurologic function score increased in the EAE group compared to that of the controls $(P<0.01)$. In addition, CAT and CK- 
BB activities significantly decreased and the calpain activity significantly increased compared with those of the control group $(P<0.05)$. The decrease of the activity of central nervous CK-BB and CAT content, as well as the increase of calpain activity at the highest time point were considered to be the consequences of EAE. Furthermore, the results revealed that use of salbutamol could alleviate disease symptoms and reduce the recurrence of the EAE disease.

Key words: Experimental autoimmune encephalomyelitis; Catalase; CK-BB; Beta 2 adrenergic agonists; Calpain

\section{INTRODUCTION}

Multiple sclerosis (MS) is an autoimmune disease characterized by inflammation-based injury of the central nervous system involving demyelination and axonal degeneration. The major pathological manifestations are infiltration by inflammatory cells along with demyelination in central nervous system white matter. Normal optic nerve and astrocytes express the beta 2 adrenergic receptor along with positive staining by brain white matter acidic protein (GFAP); however, in the cerebral white matter of patients with MS a lack of beta 2 adrenergic receptor expression on astrocytes has been identified (Jara et al., 2014). This deficit plays an important role in the mechanism of axonal damage, which involves inflammation and mitochondrial metabolism along with the production of neurotrophic factors. Accordingly, clinical studies have confirmed that beta 2 adrenergic agonists can reduce the relapse rate in patients with MS (Khoury et al., 2010). Here, through the establishment of an experimental allergic encephalomyelitis (EAE) rat model, the detection of axonal damage, and the determination of the metabolism of related enzymes in axonal mitochondria, this project aimed to reveal the effects of beta 2 adrenergic agonists on these specific aspects of MS.

Disordered mitochondrial energy metabolism has been recognized to lead to axonal degeneration during the course of MS (Su et al., 2009; Oh and O'Connor, 2013). BB isoenzyme of creatine kinase (CK-BB) is specifically expressed in astrocytes of the cerebral white matter in human and rats. Autopsies have found that compared with a normal control group, the expression level and activity of CK-BB in the brain white matter of patients with MS were significantly decreased, which might explain the higher levels of cerebral white matter phosphocreatine observed in these patients (Steen et al., 2010). Decreased levels or activities of CK-BB indicate the cell damage, as CK-BBmediated generation of ATP provides the primary energy source for mammalian brain tissue. The concentration of CK-BB in cerebral white matter is reduced by post-transcriptional and translational modification. In patients with MS, enzyme transcription might be diminished because of the lack of beta 2 adrenergic receptor expression in cerebral white matter astrocytes. ATP is substrate for serous calcium ATPase 2 (PMCA2), sarcoplasmic reticulum calcium ATPase (SERCA2), and $\mathrm{Na}^{+}$, $\mathrm{K}^{+}$-ATPase. These enzymes play key roles in the efflux of intracellular $\mathrm{Ca}^{2+}$, and their expression or activity can influence the balance of intracellular $\mathrm{Ca}^{2+}$. Elevated intracellular calcium concentration can increase calpain activation, which leads to cytoskeleton protein damage, apoptosis, and other forms of cellular damage.

In addition, cells undergo oxidative stress during the initial and developmental stages of MS. Mitochondria are the main source of cellular reactive oxygen species (Kutzelnigg et al., 2005; Press and Milbrandt, 2008). In this study, we utilized the EAE animal model to measure 
mitochondrial enzymes during the disease progression and determined the effects of therapeutic intervention with the beta 2 adrenergic agonist salbutamol. Our results suggest a new experimental basis for the clinical treatment of MS.

\section{MATERIAL AND METHODS}

\section{Experimental animals and grouping}

A total of 46 female specific pathogen free Wistar rats (Chongqing Medical University, China) weighing 180-230 g were divided randomly into different groups: the normal control group ( $\mathrm{NaCl}, 4$ rats), EAE (14 rats), dexamethasone-treated group (14 rats), and salbutamol-treated groups (14 rats). The research was sanctioned and approved by the Institutional Animal Ethics Committee, Chongqing Medical University, Chongqing, China.

\section{Establishment of the EAE animal model}

Guinea pigs weighing approximately $400 \mathrm{~g}$ were euthanized by intraperitoneal injection of an excess of $3 \%$ chloral hydrate. The spinal cord was immediately dissected and placed on ice, weighed after removal of the spinal meninges, processed with $50 \%(\mathrm{~m} / \mathrm{v})$ pre cooling saline into guinea pig spinal cord homogenate $(\mathrm{GPSCH})$ and stored at $4^{\circ} \mathrm{C}$. To generate incomplete Freund's adjuvant, $10 \mathrm{~g}$ lanolin and $40 \mathrm{~mL}$ liquid paraffin were mixed in a conical flask, autoclaved at 15 pounds of pressure per square inch (Psi) for $30 \mathrm{~min}$, and stored at $4^{\circ} \mathrm{C}$. To generate complete Freund's adjuvant (CFA), $50 \mu \mathrm{L}$ inactivated Bacillus Calmette-Guerin (National Vaccine and Serum Institute, Beijing, China) (80 mg/mL) was added into $1 \mathrm{~mL}$ incomplete Freund's adjuvant, resulting in $4 \mathrm{mg}$ tuberculosis bacilli per milliliter. Equal amounts of GPSCH and CFA were mixed and whipped with sterile syringes on ice until an emulsion was formed, which constituted the immune antigen; this was stored at $4^{\circ} \mathrm{C}$ and used within $12 \mathrm{~h}$. After intraperitoneal anesthesia with $3.5 \%$ chloral hydrate $(\mathrm{mL} / 100 \mathrm{~g}$ body weight), the Wistar rats were locally disinfected with iodine and alcohol. Each foot was injected with $0.1 \mathrm{~mL}$ immune antigen, totaling $0.4 \mathrm{~mL} /$ animal. At the same time, the hind foot back intradermal region was injected with $0.1 \mathrm{~mL}$ pertussis vaccine; the control group was co-injected with $0.1 \mathrm{~mL} 0.9 \%$ saline and CFA mixture in the same position. After the first paroxysm, animals were divided randomly into EAE, dexamethasone (DM $2 \mathrm{mg} \cdot \mathrm{kg}^{-1} \cdot \mathrm{d}^{-1}$ ) group, and salbutamol $\left(100 \mathrm{mg} \cdot \mathrm{kg}^{-1} \cdot \mathrm{d}^{-1}\right)$ groups, and the 3 groups were each divided into a remission group and a relapse group with two sub-groups according to the course of the disease; each sub-group contained 7 rats. Since our preliminary experiments suggest that as few as one or two recurrences were observed in the DM group and salbutamol group, the DM and salbutamol rats were divided into 8 and 16 days post intervention groups instead. In this experiment, the drug intervention groups were administered the intervention on the day of illness onset, and the remission and relapse times were calculated according to the number of days after disease onset.

\section{Neurologic function assessment}

The animals were observed twice daily. The daily scores for the clinical signs of EAE were assessed by taking the mean of the scores from the two observations daily. Scores were assigned on the basis of the following symptoms: 1, tail weakness; 2, tail weakness plus limb asthenia; 3 , mild limb paralysis; 4, severe limb paralysis; and 5, moribund. 


\section{Histopathological examination}

To prepare samples for histopathological examination, rats were euthanized with $3 \%$ chloral hydrate intraperitoneal anesthesia, their chest was opened, and the left ventricular space was flushed with $250 \mathrm{~mL}$ warm saline via perfusion of the ascending aorta. This was followed by reperfusion with $250 \mathrm{~mL}$ pre-cooled paraformaldehyde (40 g/L)/0.1 M phosphate buffer solution, $\mathrm{pH} 7.4$, and then the brain and spinal cord tissue were dissected out rapidly and fixed with $4 \%$ paraformaldehyde. The tissue was dehydrated by alcohol, afforded transparency by xylene, embedded in paraffin, and sliced according to standard procedures. Tissue sections were stained by hematoxylin and eosin (HE) staining.

\section{Detection of mitochondrial function}

Samples were processed as follows: approximately $100 \mathrm{mg}$ brain tissue was briefly rinsed with $1 \mathrm{~mL}$ phosphate-buffered saline (PBS), and then placed into liquid nitrogen. The next day the frozen tissue was crushed into fine powder and added to the buffer containing $20 \mathrm{mM} \mathrm{HEPES}, \mathrm{pH}$ 7.2, $215 \mathrm{mM}$ mannitol, $75 \mathrm{mM}$ sucrose, $0.1 \%$ bovine serum albumin, and $1 \mathrm{mM}$ EGTA, blended at $4^{\circ} \mathrm{C}$, and centrifuged at $10,000 \mathrm{~g}$ for $10 \mathrm{~min}$. The supernatant was harvested and stored in an $-80^{\circ} \mathrm{C}$ freezer until needed.

\section{CAT detection}

CAT activity was assessed using a CAT kit (Nanjing Maidite Corp., Nanjing, China) and measured using spectrophotometry according to the manufacturer's protocol. Briefly, $50 \mu \mathrm{L}$ of brain tissue homogenates was mixed with $50 \mu \mathrm{L}$ of $6.5 \mu \mathrm{mol} \mathrm{H}_{2} \mathrm{O}_{2}$ on a vortex mixer and incubated at $37{ }^{\circ} \mathrm{C}$ for $1 \mathrm{~min}$. $100 \mu \mathrm{L}$ of $32.4 \mathrm{mM}$ ammonium molybdate was then added to terminate the enzymatic reaction and forms a yellow complex with the remaining $\mathrm{H}_{2} \mathrm{O}_{2}$. The absorbance of the samples was then measured at $405 \mathrm{~nm}$. The catalase activity was expressed as U/mg protein. One unit of catalase activity is defined as the amount of the enzyme required to breaks down $1 \mu \mathrm{mol}$ $\mathrm{H}_{2} \mathrm{O}_{2}$ per second at $37^{\circ} \mathrm{C}$ in $1 \mathrm{mg}$ protein of brain tissue homogenate.

\section{CK-BB detection}

Enzyme-labeling and spectrophotometric determination of CK-BB activity were performed using a CK-BB kit (Shanghai Genmed Corp., Shanghai, China). Measurements were taken with a spectrophotometer: briefly, to determine the total activity, $880 \mathrm{~mL}$ GENMED buffer was placed in a new cuvette, to which was added $50 \mathrm{~mL}$ GENMED reaction liquid, and $20 \mathrm{~mL}$ GENMED substrate solution; this was incubated at $37^{\circ} \mathrm{C}$ for $3 \mathrm{~min}$, and then $50 \mathrm{~mL}$ sample was added and the 2 solutions mixed.

\section{Calpain detection}

Calpain activity was detected using a calpain kit (Shanghai Genmed Corp., Shanghai, China). The 96-well enzyme label plates were labeled as empty background contrast, standard samples, and the sample undergoing testing. Then $120 \mu \mathrm{L}$ GENMED buffer was added to all inspection wells and $50 \mu \mathrm{L}$ GENMED lysate was added to the blank background control and 
standard sample wells. The Synergy HT Multi-Mode fluorescence microplate reader (BioTek, Winooski, VT) was set to $37^{\circ} \mathrm{C}$, with an excitation wavelength of $360-380 \mathrm{~nm}$ and an emission wavelength of $440-460 \mathrm{~nm}$.

\section{Statistical methods}

SPSS 16.0 statistical software (SPSS, Chicago, IL, USA) was used for statistical analysis. The neurologic function score and pathological grading and enzyme activity data were represented as mean \pm SD (means $\pm s$ ). The comparison of measurement data between two groups with two independent samples was performed using Student's $t$-test, and correlation analysis was performed using the $r$ and $r^{2}$. $P<0.05$ was considered statistically significant, and $P<0.01$ was considered highly significantly different.

\section{RESULTS}

\section{Disease presentation in experimental rats after immunization}

Rats were observed to be depressed and exhibit loss of appetite and activity upon disease onset at approximately 12 days after immunization, which was characterized by different degrees of decreased muscle tone, limb weakness and limb paralysis that might also be associated with incontinence. Some rats exhibited restlessness, irritability, and aggressive behavior. About 8 days after disease onset all illness remitted, and most rats were restored to normal, although a few animals had no obvious remission. About 16 days after the first signs of disease onset, some animals exhibited a repeat of the paralysis symptom. Compared with the EAE group, the animals in the drug intervention group had obviously alleviated disease symptoms with less recurrence. There were 7 instances of animal recurrence from among the 14 animals belonging to the EAE group; the recurrence rate was thus 50\%. However, there was 1 animal recurrence from among the 14 animals belonging to salbutamol group; this recurrence rate was $7.1 \%$. There were 2 instances of recurrence out of 14 animals in the DM group; the recurrence rate for this group was $14.2 \%$. The recurrence rate of the EAE group was obviously higher than those of the DM and salbutamol groups; the differences were statistically significant $(P<0.05)$. There was no significant difference between the salbutamol and DM groups $(P>0.05)$.

Comparison of the neurologic function score between each group showed that at 8 days, the average score of neurologic function for the EAE, salbutamol, and DM groups were $1.70 \pm$ $0.05,0.20 \pm 0.045$, and $0.20 \pm 0.055$ points, respectively. The neurologic function score of the EAE group was significantly higher than those of the salbutamol and DM groups $(P<0.05)$ (Figure 1). However, the neurologic function score between the salbutamol and DM groups were not significantly different $(P>0.05)$. For rats at 16 days, the average scores of neurologic function for the EAE, salbutamol, and DM groups were $4 \pm 0.61,1.50 \pm 0.55$, and $1.80 \pm 0.48$ points, respectively. The neurologic function score of the EAE group was significantly higher than those of the salbutamol and DM groups $(P<0.05)$ (Figure 1), whereas the scores between the salbutamol and DM groups had no statistically significant difference.

\section{Histopathological examination}

Following HE staining, no infiltration of inflammatory cells were observed in spinal cord 
tissue in the normal control group, but there was a wide range of inflammatory cells such as mononuclear cells and diffuse lymphoid cell infiltration in the spinal cord tissue of the disease groups. "Cuffing" formations of the perivascular inflammatory cells between the gray and white matter of the spinal cord were also observed (Figure 2A).

Visible diffuse inflammatory cell perivascular infiltrates composed of mononuclear and lymphoid cells in the spinal cord tissue of the remission (Figure 2B) and relapse groups (Figure 2C) were also observed. However, visualization of "cuffing" formations was rare. In contrast, the number of inflammatory cells in tissues of the drug intervention group was obviously reduced.

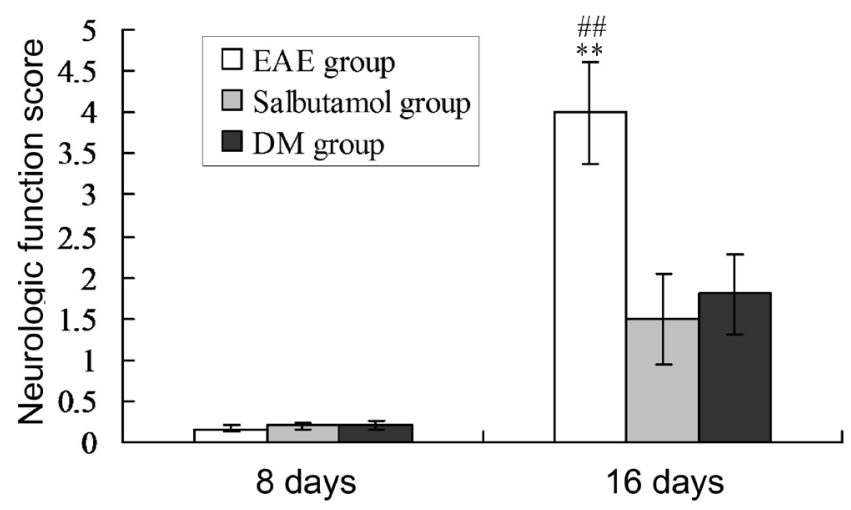

Figure 1. Neurologic function score in the EAE group compared with the control group. Neurologic function score was plotteas mean \pm standard error. ${ }^{* *} P<0.01$, ${ }^{\# P} P 0.01$ represent the neurologic function score compared with the salbutamol and DM groups, respectively. EAE, experimental allergic encephalomyelitis; DM, dexamethasone.
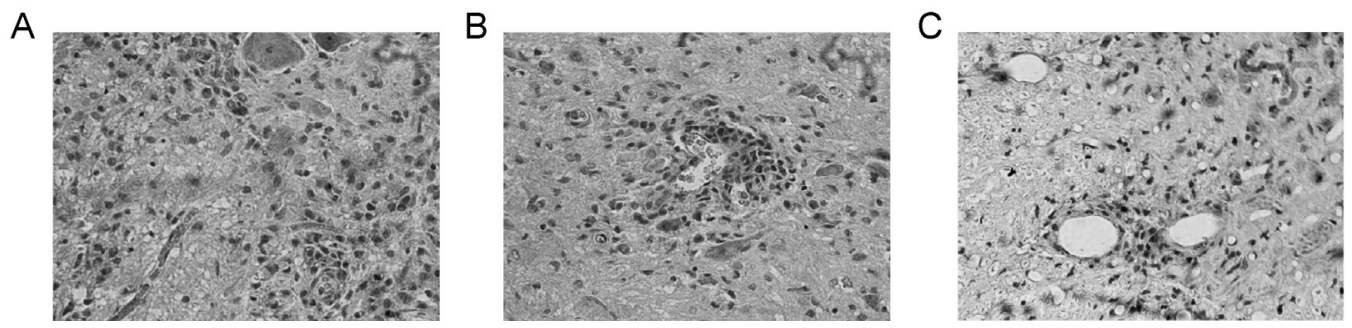

Figure 2. Infiltration of inflammatory cells and perivascular cuffing in the spinal cord of diseased EAE rats (HE 200X), A) At the crest-time stage; B) remission stage; and (C) relapse stage. EAE, experimental allergic encephalomyelitis; $\mathrm{HE}$, hematoxylin and eosin.

\section{Mitochondria CAT content in spinal cord tissue}

The average content of CAT in the EAE, DM, and salbutamol groups at 8 days (remission) was $210.88 \pm 2.14,273.65 \pm 6.18$, and $308.12 \pm 5.57 \mathrm{U} / \mathrm{mg}$ protein, respectively. The CAT level in EAE group compared with the salbutamol and DM groups were highly significantly different $(P<$ 0.01) (Figure $3 A$ ). For rats at 16 days (relapse), the average content of CAT in the EAE, DM, and salbutamol groups was $159.20 \pm 2.83,180.35 \pm 3.12$, and $186.12 \pm 2.55 \mathrm{U} / \mathrm{mg}$ protein, respectively. The CAT level in the EAE group was significantly different from those of the salbutamol and DM groups $(P<0.05)$ (Figure $3 A)$. No statistically significant difference was observed between the DM and salbutamol groups $(P>0.05)$. 
A

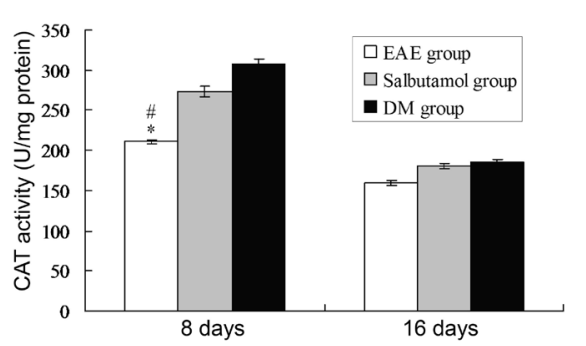

C

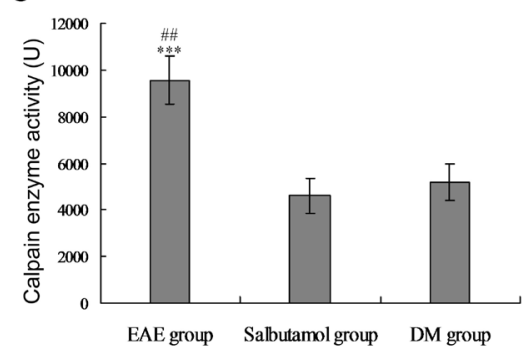

B

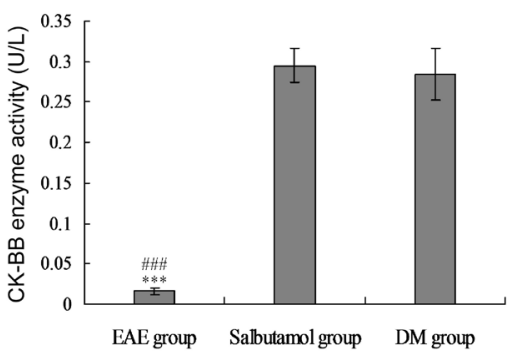

Figure 3. Activity of CAT, CK-BB and calpain in the EAE group. A) CAT B) CK-BB and C) calpain were plotted as mean \pm standard error. ${ }^{*} \mathrm{P}<0.05$ and ${ }^{~} \mathrm{P}<0.05$ represent CAT activity compared with the salbutamol and DM groups, respectively. ${ }^{* * *} \mathrm{P}<0.001$ represents the activities of CK-BB and calpain compared with the control group, respectively. ${ }^{\#} \mathrm{P}<0.001$ represents the CK-BB activity compared with that of the control group. ${ }^{* *} \mathrm{P}<0.01$ represents the calpain activity compared with that of the control group. CAT, catalase; CK-BB, creatine kinase BB isoenzyme; $\mathrm{EAE}$, experimental allergic encephalomyelitis; DM, dexamethasone.

\section{Activities of CK-BB and calpain in the EAE group compared with the control group}

The CK-BB activity in the brain tissues of the EAE group was reduced significantly compared to that of the control group $(P<0.05)$ (Figure $3 B$ ). In contrast, the calpain activity in the EAE group was significantly increased compared with that of the control group $(P<0.05)$ (Figure $3 C)$.

\section{Correlation analysis}

The correlation analysis results indicated that the neurologic function correlated positively with the pathological grading in rats $\left(r=0.516, r^{2}=0.266, P<0.01\right)$ (Figure 4A). The higher the neurological function score, the more obvious were the pathological changes in the diseased rats. This result shows that the neural function scores and pathological changes are closely correlated.

The correlation analysis also showed that the neurologic function was negatively correlated with the CK-BB enzyme activity $\left(r=-0.812, r^{2}=0.659, P<0.01\right)$ (Figure 4B). In other words, the higher the neurological function score, the lower the CK-BB activity. The result showed that there is a close albeit inverse correlation between the neurologic function score and CK-BB enzyme activity.

The results also indicated that neurologic function was positively correlated with calpain enzyme activity $\left(r=0.864, r^{2}=0.746, P<0.01\right)$ (Figure $\left.4 C\right)$. As for the pathologic grading, the higher neurological function scores were associated with higher calpain activities. The result showed that the neurologic function score and calpain activity had a close correlation. 
A

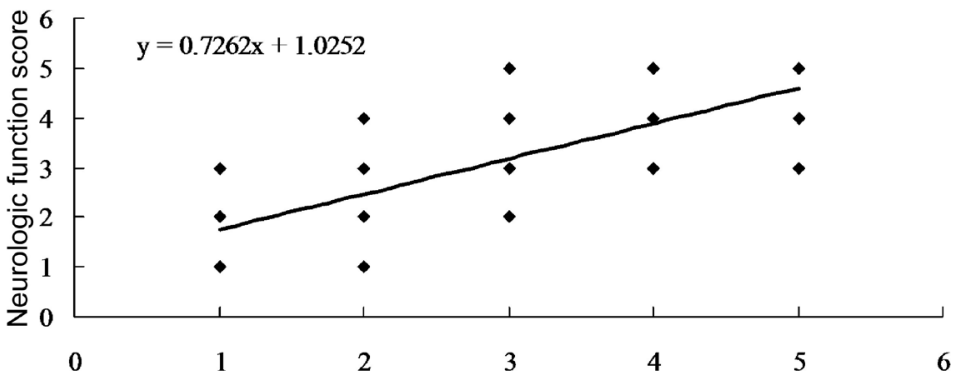

B

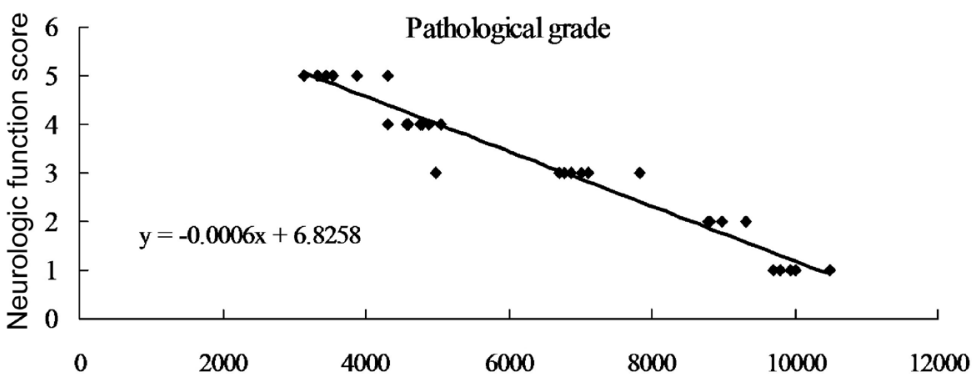

C

CK-BB enzyme activity

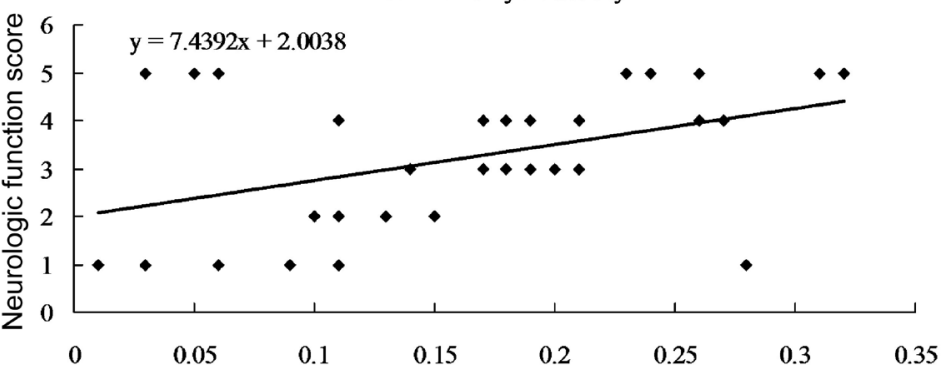

Calpain enzyme activity

Figure 4. Correlation between neurologic function grade and pathological grade, CK-BB enzyme activity, and calpain enzyme activity. A) Correlation between neurologic function grade and pathological grade; B) CK-BB enzyme activity; and C) calpain enzyme activity. CK-BB, creatine kinase BB isoenzyme.

\section{DISCUSSION}

The primary consequences of a lack of beta 2 adrenergic receptor on astrocytes during the pathophysiological process of MS are as follows: 1) collapse of the myelin antigen tolerance mechanism, leading to T cell-mediated inflammatory demyelinating lesions (Kornek et al., 2000); 2) axonal degeneration caused by advanced physical function disabilities; and 3) a lack of neurotrophic factors that causes oligodendrocyte damage and leads to damage of the myelin sheath and axons (Frischer et al., 2009). During the MS progressive stage, widespread damage appears across the brain and spinal cord, although the degree of axonal loss differs between individuals with lesions, with an average loss of approximately $60-70 \%$ compared with those with non-lesioned axons. In addition, the normal appearance of the white matter itself also exhibits advanced axon damage and 
loss at this stage (DeLuca et al., 2000). The results of pathologic analysis of axonal injury are closely related to the level of neural function; when axonal damage reaches a certain threshold value (more than $30 \%$ of axons), patients can exhibit clinical symptoms of neurologic function deficiency.

Adrenergic mechanisms play an important role in the regulation of immune response. It has been reported that oral administration of salbutamol, a beta 2 adrenergic agonist, can significantly reduce interleukin-12 and gamma interferon levels in patients with MS (Makhlouf et al., 2001). CAT can clear away the primary oxidation products such as $\mathrm{H}_{2} \mathrm{O}_{2}$, reducing their oxidative damage abilities (Tolman and Dalpiaz, 2007; Lee et al., 2009). Increased expression of CAT in the mitochondria is taken as evidence of improvement of mitochondrial anti-oxidative stress levels. CK-BB provides the main source of energy for mammalian brain tissue. In this study, we demonstrated that compared with the control group, the CK-BB enzyme activity in EAE rats was markedly decreased, indicating that EAE model rats exhibit a failure of their primary energy resource. Correlation analysis between CK-BB enzyme activity and neurologic function showed that they were closely related, with higher neurologic function scores associated with lower enzyme activities. This suggests that the more severe the EAE disease, the more the energy failure of the central nervous tissue in rats becomes obvious. From this experiment, we have also found that the activity of calpain in the EAE group significantly increased compared with that of the control group. Furthermore, we identified that the enzyme activity and neural function score were also closely related, such that higher neural function scores were associated with higher enzyme activities, suggesting that in severe EAE rats, the increased activity of calpain would increase the $\mathrm{Ca}^{2+}$ concentration inside the cell, triggering cell apoptosis or cytoskeleton damage.

In this study, we applied salbutamol as a therapeutic intervention for the EAE model, and observed the pathologies of the brain and spinal cord tissue, gauged the activities of CK-BB and calpain and detected the content of CAT in the mitochondria, graded the neurologic function score of $E A E$ rats after the intervention, and evaluated the recurrence rates. All of our results confirmed that salbutamol could obviously reduce the neurologic damage of EAE model rats, improve the neurologic symptoms, and reduce the recurrence rate of EAE. These findings might provide guidance for the future prevention and treatment of MS, and suggest that the mitochondria could be used as a treatment target for MS. This research provides clues for the development of new drugs to treat EAE; however, additional intensive basic and clinical research needs to be performed for confirmation of our results.

\section{Conflicts of interest}

The authors declare no conflict of interest.

\section{ACKNOWLEDGMENTS}

Research supported by a Natural Science Foundation Project of the Chongqing Municipal Chongqing Science and Technology Commission (\#CQ CSTC) (\#cstc2012jjA10127).

\section{REFERENCES}

DeLuca GC, Ebers GC and Esiri MM (2000). Axonal loss in multiple sclerosis: a pathological survey of the corticospinal and sensory tracts. Brain 127: 1009-1018.

Frischer JM, Bramow S, Dal-Bianco A, Lucchinetti CF, et al. (2009). The relation between inflammation and neurodegeneration in multiple sclerosis brains. Brain 132: 1175-1189. 
Jara M, Sidovar MF and Henney HR 3rd (2014). Prescriber utilization of dalfampridine extended release tables in multiple sclerosis: a retrospective pharmacy and medical claims analysis. Ther. Clin. Risk Manag. 11: 1-7.

Khoury SJ, Healy BC, Kivisäkk P, Viglietta V, et al. (2010). A randomized controlled double-masked trial of albuterol add-on therapy in patients with multiple sclerosis. Arch. Neurol. 67: 1055-1061.

Kornek B, Storch MK, Weissert R, Wallstroem E, et al. (2000). Multiple sclerosis and chronic autoimmune encephalomyelitis: a comparative quantitative study of axonal injury in active, inactive, and remyelinated lesions. Am. J. Pathol. 157: 267-276.

Kutzelnigg A, Lucchinetti CF, Stadelmann C, Brück W, et al. (2005). Cortical demyelination and diffuse white matter injury in multiple sclerosis. Brain 128: 2705-2712.

Lee BW, Kwon SJ, Chae HY, Kang JG, et al. (2009). Dose related cytoprotective effect of alpha-lipoic acid on hydrogen peroxide-induced oxidative stress to pancreatic beta cells. Free Radic. Res. 43: 68-77.

Makhlouf K, Comabella M, Imitola J, Weiner HL, et al. (2001). Oral salbutamol decreases IL-12 in patients with secondary progressive multiple sclerosis. J. Neuroimmunol. 117: 156-165.

Oh J and O'Connor PW (2013). An update of teriflunomide of treatment of multiple sclerosis. Ther. Clin. Risk Manag. 9: 177-190.

Press C and Milbrandt J. (2008). Nmnat delays axonal degeneration caused by mitochondrial and oxidative stress. J. Neurosci. 28: 4861-4871.

Steen C, Wilczak N, Hoogduin JM, Koch M, et al. (2010). Reduced creatine kinase B activity in multiple sclerosis normal appearing white matter. PLoS One 5: e10811.

Su KG, Banker G, Bourdette D and Forte M (2009). Axonal degeneration in multiple sclerosis: the mitochondrial hypothesis. Curr. Neurol. Neurosci. Rep. 9: 411-417.

Tolman KG and Dalpiaz AS (2007). Treatment of non-alcoholic fatty liver disease. Ther. Clin. Risk Manag. 3: 1153-1163. 